\section{Avaliação da cultura de segurança do doente em ci- rurgia ambulatória pelos enfermeiros: protocolo de scoping review}

Nurses' assessment of patient safety culture in ambulatory surgery: scoping review protocol

Evaluación de la cultura de seguridad del paciente en la cirugía ambulatoria por parte de los enfermeros: protocolo de scoping review

Joana Raquel Luís Pinto ${ }^{1}$

(iD) https://orcid.org/0000-0003-1095-0088

Ana Cristina Rafael Matias ${ }^{2}$

(D) https://orcid.org/0000-0001-5601-3132

Luís Leitåo Sarnadas ${ }^{3}$

(iD) https://orcid.org/0000-0002-7022-7062

${ }^{1}$ Universidade Católica Portuguesa, Porto, Portugal; Hospital da Luz Coimbra, Coimbra, Portugal

${ }^{2}$ Hospital Distrital De Leiria, Leiria, Portugal

${ }^{3}$ Unidade de Investigaçăo em Ciências da Saúde: Enfermagem, Escola Superior de Enfermagem de Coimbra, Coimbra, Portugal

Autor de correspondência

Joana Raquel Luís Pinto

E-mail: joanaraqp@esenfc.pt

Recebido: 05.05 .20

Aceite: 02.09 .20

\section{Resumo}

Enquadramento: A enfermagem perioperatória tem por base uma abordagem dinâmica e global do doente, transversal à cirurgia ambulatória. Associada a este paradigma e no sentido de avaliar a qualidade dos cuidados de enfermagem e a segurança dos mesmos, é pertinente considerar a cultura de segurança do doente.

Objetivo: Mapear a evidência disponível sobre a avaliação da cultura de segurança do doente em cirurgia de ambulatório pelos enfermeiros.

Método de revisáo: Metodologia proposta pelo Joanna Briggs Institute. A estratégia de pesquisa será adequada a cada base de dados/repositório na identificação de estudos relevantes. Proceder-se-á à anácluídos serão alvo de extração de dados. Este processo será executado por dois revisores independentes. Apresentaçáo e interpretaçáo dos resultados: Os resultados serão apresentados de forma narrativa e de tabelas.

Conclusáo: Esta scoping review contribuirá para identificar a perspetiva dos enfermeiros sobre a cultura de segurança do doente em cirurgia de ambulatório, reforçando o potencial impacto na segurança dos cuidados de enfermagem.

Palavras-chave: procedimentos cirúrgicos ambulatórios; segurança do paciente; gestão da segurança; enfermagem; revisão

\section{Abstract}

Background: Perioperative nursing is based on a dynamic and holistic approach to the patient that extends to ambulatory surgery. With this paradigm, it is essential to consider patient safety culture in the assessment of the quality and safety of nursing care.

Objective: To map the evidence on nurses' assessment of patient safety culture in ambulatory surgery. Review method: Methodology proposed by the Joanna Briggs Institute. The search strategy will be adapted to each database/repository to identify relevant studies. Titles and abstracts will be screened, and those that do not meet the inclusion criteria will be excluded. The included articles will be subject to data extraction by two independent reviewers.

Presentation and interpretation of results: The results will be presented in narrative and tabular form. Conclusion: This scoping review will contribute to identifying nurses' perspectives on patient safety culture in ambulatory surgery, reinforcing the potential impact on the safety of nursing care.

Keywords: ambulatory surgical procedures; patient safety; safety management; nursing; review

Resumen

Marco contextual: La enfermería perioperatoria se basa en un enfoque dinámico y global del paciente, transversal a la cirugía ambulatoria. Asociada a este paradigma y con el objetivo de evaluar la calidad de la atención de enfermería y la seguridad, es pertinente considerar la cultura de seguridad del paciente.

Objetivo: Mapear las pruebas disponibles sobre la evaluación de la cultura de seguridad del paciente en la cirugía ambulatoria por parte de los enfermeros.

Método de revisión: Metodología propuesta por el Instituto Joanna Briggs. La estrategia de investigación será adecuada a la identificación de los estudios relevantes en cada base de datos/repositorio. Se procederá a analizar los títulos y resúmenes, excluyendo los que no presenten los criterios indicados. Los artículos incluidos estarán sujetos a la extracción de datos. Este proceso será realizado por dos revisores independientes.

Presentación e interpretación de los resultados: Los resultados se presentarán en forma narrativa y en tablas.

Conclusión: Esta revisión exploratoria (scoping review) contribuirá a identificar la perspectiva de los enfermeros sobre la cultura de seguridad del paciente en la cirugía ambulatoria, lo que reforzará el potencial impacto en la seguridad de la atención de enfermería.

Palabras clave: procedimientos quirúrgicos ambulatorios; seguridad del paciente; gestión de la seguridad; enfermería; revisión lise de títulos e resumos, excluindo aqueles que não apresentem os critérios delineados. Os artigos in-

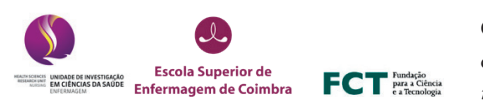

Como citar este artigo: Pinto, J. R., Matias, A. C., \& Sarnadas, L. L. (2020). Avaliaçāo da cultura de segurança do doente em cirurgia ambulatória pelos enfermeiros: protocolo de scoping review. Revista de Enfermagem Referência, 5(4), e20059. doi:10.12707/RV20059 


\section{Introdução}

A cirurgia de ambulatório (CA) é um modelo organizativo de prestação de cuidados cirúrgicos, por excelência centrado no doente, sendo por isso relevante para a melhoria da eficiência, efetividade e qualidade dos cuidados de saúde e organização hospitalar (Despacho n. ${ }^{\circ}$ 1380/2018; Pinto \& Sarnadas, 2020).

Neste regime cirúrgico, após o doente ser admitido na instituição hospitalar, é realizada a intervenção cirúrgica programada e este tem alta num período inferior a 24 horas. A CA tem múltiplas vantagens internacionalmente reconhecidas em relação à cirurgia eletiva com internamento. A nível organizacional permite uma reorganização de toda a estrutura hospitalar, por exemplo, através da utilização do internamento para situações mais complexas, assim como redução do tempo de espera para cirurgia e criação de programas centrados no doente. Por outro lado, apresenta vantagens económicas diretas através da redução dos custos hospitalares e indiretas pela menor morbilidade que lhe está associada. À CA estão associadas menores taxas de complicaçôes pós-operatórias e menor probabilidade de infeçóes associadas aos cuidados de saúde. Ao nível social, a realização de procedimentos cirúrgicos em ambulatório apresenta um impacto muito positivo para o doente pois este pode recuperar em ambiente familiar, havendo uma menor rutura sociofamiliar, ao mesmo tempo que permite uma integração socioprofissional mais célere (Comissão Nacional Para o Desenvolvimento da Cirurgia de Ambulatório, 2008; Despacho n. ${ }^{0}$ 1380/2018; Pinto \& Sarnadas, 2020).

A prática da CA tem sofrido um forte crescimento neste início de século a nível mundial e nacional. Portugal constitui-se como um exemplo de referência ao nível da expansáo da CA. No ano de 2000 apenas $10 \%$ do total de cirurgias ambulatorizáveis eram realizadas neste regime e em 2006 pouco mais de um quarto da produção cirúrgica nacional foi realizada em ambulatório. Atualmente, mais de $83 \%$ do total de cirurgias programadas realizadas no nosso país decorrem em regime de ambulatório (Administração Central do Sistema de Saúde, 2017; Despacho n. ${ }^{\circ}$ 1380/2018; Serviço Nacional de Saúde, 2020).

Este desenvolvimento da CA nas últimas décadas em Portugal deve-se essencialmente aos incentivos e às orientaçôes institucionais e governamentais desenvolvidas (Pinto \& Sarnadas, 2020). Tendo por base o perfil inovador da CA em relação à cirurgia eletiva com internamento e com o propósito de maximizar as suas vantagens, é necessário realizar um "diagnóstico da situação, identificando áreas que, apesar do crescimento, carecem ainda de desenvolvimento" (Despacho n. ${ }^{\circ} 1380 / 2018$, p. 4511). Assim, com o Despacho n. ${ }^{\circ} 1380 / 2018$, de 8 de fevereiro, o Governo estabeleceu a criação de um grupo de trabalho para o acompanhamento do desenvolvimento da CA, com o objetivo de realizar a sua avaliaçáo nos últimos 10 anos em Portugal, identificando eventuais constrangimentos ao seu crescimento (estruturais, de recursos humanos, de formação ou outros).

Alguma literatura internacional aponta possíveis constrangimentos ao desenvolvimento da CA. Por exemplo, apesar de mais de dois terços da produção cirúrgica nos Estados Unidos da América, o que corresponde, anualmente, a mais de 25 milhóes de cirurgias, serem realizada em regime de ambulatório, pouco se sabe sobre segurança do doente e qualidade dos cuidados neste contexto (Aston, 2014; Molina et al., 2017; Nelson, 2018).

A segurança do doente, como referem Pinto e Sarnadas (2020), é um indicador relevante da qualidade dos cuidados de saúde e a promoção de uma cultura de segurança nos ambientes de prestação de cuidados é, atualmente, um meio para a sua obtenção e eventual melhoria.

A cultura de segurança de uma organização de saúde é um conceito transversal desde a liderança administrativa até aos prestadores de cuidados, e esta pode ser definida como o conjunto dos valores individuais e de grupo, das suas atitudes, perceçóes, competências e comportamentos que determinam o seu empenho e compromisso para a prosperidade organizacional e a sua gestão segura (Smith, Sorra, Franklin, Rockville, \& Behm, 2015). Esta assenta na premissa de que competências não técnicas como o trabalho em equipa, comunicação e reporte de eventos adversos são essenciais para uma prática de cuidados de saúde de qualidade e segura, sabendo que defeitos nestes aspetos podem lesar os doentes, tanto como os erros técnicos. Assim, um estilo de comunicação baseado na confiança mútua, perceções partilhadas sobre a importância da segurança e a aposta na eficácia de medidas preventivas são características de organizaçóes com uma cultura de segurança positiva (Attree \& Newhold, 2009; Fan et al., 2016; Smith, Sorra, Franklin, Rockville, \& Behm, 2015). Tendo em conta o fenómeno de expansão mundial da CA, a Agency for Healthcare Research and Quality (AHRQ) desenvolveu, em 2014, um questionário intitulado Ambulatory Surgery Center Survey on Patient Safety Culture (Smith et al., 2015) especificamente para Unidades de Cirurgia de Ambulatório (UCAs) e avalia várias dimensóes da Cultura de Segurança do Doente (CSD), nomeadamente a comunicação relacionada com informação do doente, abertura comunicacional, equipa, pressão e ritmo de trabalho, trabalho em equipa, conhecimento dos profissionais das tarefas a executar, aprendizagem organizacional com vista à melhoria contínua, resposta ao erro e apoio que os administradores/ superiores hierárquicos dão para a segurança do doente. Este questionário encontra-se traduzido e validado para a populaçáa portuguesa por Pinto e Sarnadas (2020).

O objetivo da avaliaçáo da CSD é permitir às organizaçóes conhecer as características da sua cultura de segurança, identificando os seus pontos fortes e áreas a melhorar, sensibilizar os profissionais, criar planos de intervenção e realizar benchmarking dos resultados (Pinto \& Sarnadas, 2020; Smith et al., 2015).

A enfermagem perioperatória em contexto de CA é, então, recente e tem forte influência na forma como se prestam cuidados de saúde aos doentes cirúrgicos (Davidson, 2014). De facto, a tendência crescente das cirurgias realizadas em regime de ambulatório quer a nível mundial, quer em Portugal, confirmam que mais e melhores cuidados de enfermagem serão necessários neste contexto (Davidson, 2014).

O papel do enfermeiro é indubitavelmente de relevo, uma 
vez que a sua presença é transversal em todos os momentos da CA. O enfermeiro depara-se com desafios únicos e assume um papel que ultrapassa a gestão e prestação de cuidados: encontra-se numa posição em que tem de assegurar que todos os procedimentos e atividades são realizadas em segurança, ao mesmo tempo que acompanha o doente desde a sua entrada até ao momento da alta (Davidson, 2014; Nelson, 2018).

O conhecimento da forma como os enfermeiros avaliam a CSD na UCA onde desenvolvem a sua atividade profissional poderá, assim, ter implicaçóes profundas ao nível da liderança direta, assim como a nível da liderança de topo, concorrendo para um melhor ambiente de prática de cuidados perioperatórios.

Foi realizada uma pesquisa preliminar nas bases de dados JBI Database of Systematic Reviews and Implementation Reports, Cochrane Central Register of Controlled Trials, CINAHL, MEDLINE e PROSPERO, não tendo sido identificada nenhuma revisão de literatura no âmbito desta temática. Assim, os autores consideraram pertinente a realização de uma scoping review, tendo por base as orientaçóes metodológicas sugeridas pelo Joanna Briggs Institute (Peters et al., 2020).

Esta scoping review tem como objetivo mapear a evidência existente relativa à avaliação da cultura de segurança do doente em cirurgia de ambulatório pelos enfermeiros. Mais especificamente, com esta revisão pretende-se dar resposta à seguinte questão de investigação: Como avaliam os enfermeiros a cultura de segurança do doente em cirurgia de ambulatório?

\section{Método de revisão sistemática}

A opção pela realização de uma scoping review teve por base o principal objetivo deste tipo de revisão de literatura: mapear os conceitos-chave nos quais se baseia uma determinada área de conhecimento. Assim, com a scoping review pretende-se identificar quais os tipos de evidência disponível em áreas de conhecimento específicas, fornecendo uma visão geral e ampla do que já foi estudado, identificar e analisar lacunas de investigação existentes e justificar outros trabalhos científicos mais específicos na área em estudo, como por exemplo revisões sistemáticas de literatura (Peters et al., 2020).

\section{Estratégia de pesquisa e identificação dos estudos}

A scoping review proposta será conduzida de acordo com a metodologia proposta pelo Joanna Briggs Institute, identificando critérios de inclusão e exclusão para Participantes, Conceito e Contexto (PCC), tendo em conta os componentes da questão de investigação. Assim sendo, esta revisão considerará estudos que incluam como participantes enfermeiros a exercer funções em unidades de cirurgia de ambulatório, de instituições públicas ou privadas independentemente do seu vínculo laboral, idade e tempo de profissáo.

Relativamente ao conceito serão considerados estudos que avaliem a cultura de segurança do doente em unidades de cirurgia ambulatória e as dimensóes que a constituem, tendo em vista a identificação de áreas problemáticas assim como implicaçóes que possam ter na segurança do doente. Quanto ao contexto, serão incluídos estudos realizados em unidades de cirurgia ambulatória, integradas ou não em hospitais, públicas ou privadas.

Esta scoping review incluirá estudos quantitativos, qualitativos e de métodos mistos. Serão considerados estudos primários, assim como revisões sistemáticas, dissertações, textos de opinião e literatura cinzenta, de acordo com as três etapas definidas para scoping reviews pelo Joanna Briggs Institute (Peters et al., 2020). Não serão considerados limites temporais geográficos ou culturais, tendo em conta que se pretende um entendimento de como a CSD é investigada e percebida em diversos contextos culturais. Na primeira será realizada uma pesquisa inicial limitada para identificar artigos sobre o tema em questáo nos motores de busca PUBMED e EBSCO com interface para as bases de dados CINAHL Complete, MEDLINE Complete, Nursing \& Allied Health Collection: Comprehensive; Cochrane Central Register of Controlled Trials e MedicLatina, recorrendo aos descritores MESH 'safety culture; organizational culture; safety management' 'patient safety'; 'ambulatory surgery'; 'outpatient surgery' 'ambulatory surgical procedures'; 'nursing'. Serão analisados os títulos, os resumos e os termos de indexação dos resultados selecionados, de forma a identificar termos e palavras-chave alternativos e específicos. Na segunda etapa, os termos de indexação e palavras-chave identificados serão utilizados para a realização de nova pesquisa nos motores de busca e bases de dados já identificados, pesquisando também de forma conjugada com linguagem natural. Na terceira etapa, serão analisadas as referências bibliográficas de todas as publicações selecionadas para identificação de fontes de informação adicionais.

Caso haja necessidade de algum esclarecimento adicional ao que está publicado, os revisores procurarão entrar em contacto com os autores do material em análise.

Após a pesquisa, a seleção dos resultados relevantes iniciar-se-á com a análise dos títulos e resumos, com base nos critérios de inclusão para a revisão já descritos e por dois revisores independentes. O texto completo dos estudos selecionados será avaliado em detalhe com base nos critérios de inclusão. Os motivos da exclusão de estudos, após análise do texto integral, que não atendam aos critérios de inclusão serão registados e relatados na scoping review. Quaisquer divergências que surjam entre os revisores serão resolvidas por meio de discussão e consenso ou com recurso a um terceiro revisor. Não será realizada a avaliação da qualidade metodológica dos estudos incluídos por se tratar de uma scoping review (Peters et al., 2020).

O processo de pesquisa será apresentado na íntegra sob forma narrativa e esquemática na scoping review, através de um diagrama de fluxo (PRISMA), detalhando a forma como a pesquisa foi realizada, seleção dos resultados utilizados, eliminação de duplicados e o que foi acrescentado com a terceira etapa da pesquisa (Liberati et al., 2009; Peters et al., 2020).

Todas as referências bibliográficas identificadas serão agrupadas e geridas no software Mendeley 1.19.4 /2019 (Mendeley Ltd., Elsevier, Holanda). 


\section{Extraçáo de dados}

A extração dos dados para a scoping review será realizada por dois revisores independentes, após confirmação da pertinência das publicaçóes previamente selecionadas. Esta será aferida pela utilização de um instrumento de extração de dados desenvolvido pelos investigadores, alinhado com o objetivo e questáo de revisão (Figura 1). $\mathrm{O}$ instrumento que se apresenta é preliminar, podendo sofrer alteraçóes pertinentes, mediante as necessidades resultantes da leitura e análise das publicaçóes elegíveis. Para que os investigadores se familiarizem com o instrumento de extraçáo de dados, será realizado um teste piloto com o número necessário de publicaçôes.

Caso seja necessário algum esclarecimento adicional durante este processo, os autores das publicaçóes em análise poderão ser contactados.

\begin{tabular}{|c|c|}
\hline $\begin{array}{l}\text { Titulo da Revisão } \\
\text { Avaliação da Cultura de Segurança do Doente em } \\
\text { cirurgia ambulatória pelos enfermeiros: protocolo de } \\
\text { scoping review }\end{array}$ & $\begin{array}{l}\text { Responsável pela } \\
\text { extração: }\end{array}$ \\
\hline \multicolumn{2}{|c|}{$\begin{array}{l}\text { Questão de revisão: } \\
\text { Como avaliam os enfermeiros a cultura de segurança do doente em cirurgia de } \\
\text { ambulatório? }\end{array}$} \\
\hline \multicolumn{2}{|c|}{ Detalhes e características } \\
\hline $\begin{array}{l}\text { Dados bibliográficos } \\
\text { Autor(es); ano de } \\
\text { publicaçâo; título; } \\
\text { periódico onde foi } \\
\text { publicado; país de } \\
\text { origem }\end{array}$ & \\
\hline $\begin{array}{l}\text { Tipo de estudo; } \\
\text { Objetivos; População e } \\
\text { amostra }\end{array}$ & \\
\hline $\begin{array}{l}\text { Método de colheita de } \\
\text { dados }\end{array}$ & \\
\hline $\begin{array}{l}\text { Resultados } \\
\text { encontrados } \\
\text { Dimensões da CSD } \\
\text { avaliadas } \\
\text { Conceitos para dar } \\
\text { resposta à questão de } \\
\text { revisão }\end{array}$ & \\
\hline $\begin{array}{l}\text { Referências } \\
\text { bibliográficas } \\
\text { encontradas }\end{array}$ & \\
\hline
\end{tabular}

\section{Síntese dos dados}

Os dados extraídos das publicaçôes incluídas na revisão serão apresentados de forma narrativa, prevendo-se o recurso a tabelas e/ou figuras. Esta síntese será alinhada com o âmbito e objetivo da scoping review. Este processo terá por base o consenso de dois revisores independentes e o apoio de um terceiro revisor para resolver alguma divergência.

\section{Apresentaçáo e interpretaçáo dos resultados}

Os dados recolhidos serão apresentados em forma narrativa e, eventualmente, em tabela ou esquema, por forma a mapear a evidência disponível relativa à avaliação da CSD no âmbito de CA pelos enfermeiros. Serão também apresentados quadros resumo dos artigos incluídos na revisão. A identificação, caracterização e síntese do conhecimento nesta área procurará estar alinhada com o objetivo e questáo de revisão que se propóe.

A scoping review que se propóe, irá contribuir para a disseminação do conhecimento numa área emergente na enfermagem perioperatória e com grande impacto em todo o mundo. Por outro lado, dotará de elementos importantes e valiosos para tomadas de decisão enfermeiros com funçốes de liderança e/ou gestáo de UCAs, assim como a gestão intermédia e de topo destas unidades, de aspetos que contribuem para uma melhor segurança dos doentes na sua organizaçáo e de outros que devem ser melhorados, de forma a criar uma CSD a nível organizacional que promova melhores e mais seguros cuidados de enfermagem.

\section{Conclusão}

Tendo em consideração todas as vantagens que estão associadas à CA, o seu crescimento marcado a nível mundial e nacional, assim como a necessidade de prestar cuidados de enfermagem neste contexto cirúrgico assentes na segurança e na qualidade, a avaliaçáo da CSD do doente em CA é um imperativo. Perceber quais os pontos fortes e os aspetos que podem ser impulsionadores de uma melhoria da cultura de segurança neste ambiente de prática de 
enfermagem tâo complexo, é urgente. Espera-se que os contributos para a prática clínica desta scoping review sejam evidentes e marcados, pois poderão ser úteis na criação de intervençóes de melhoria organizacional nas UCAs. Ao nível da investigação futura, espera-se que esta scoping review constitua um primeiro exercício que fundamente a elaboração de questôes mais específicas para a realização de uma revisão sistemática da literatura.

\section{Contribuiçáo de autores}

Conceptualização: Pinto, J. R., Matias, A. C., Sarnadas, L. L.

Metodologia: Pinto, J. R., Matias, A. C.

Supervisão: Pinto, J. R., Sarnadas, L. L.

Redação - preparação do rascunho original: Pinto, J. R., Matias, A. C.

Redação - revisão e edição: Pinto, J. R.

\section{Referências bibliográficas}

Administração Central do Sistema de Saúde. (2017). Atividade cirúrgica do SNS atinge o valor mais elevado de sempre em 2016. Recuperado de http://www.acss.min-saude.pt/2017/08/29/atividade-cirurgica-do-sns-atinge-o-valor-mais-elevado-de-sempre-em-2016/

Aston, G. (2014). Measuring quality and safety in outpatient surgery. Hospitals \& Health Networks, 88(9),1068-8838, pp. 34-37.

Attree, M., \& Newbold, D. (2009). Risk, safety, and reliability. From cult to culture? Journal of Nursing Management, 17(2), 145-150. doi:10.1111/j.1365-2834.2009.01000.x/epdf

Comissão Nacional para o Desenvolvimento da Cirurgia Ambulatório. (2008). Relatório final: Cirurgia de ambulatório: Um modelo de qualidade centrado no utente. Recuperado de http://www.apca. com.pt/documentos/relatorio_final_CNADCA_20Out08.pdf

Davidson, J. (2014). Why a column on ambulatory best practices? AORN Journal, 99(5), 612-615. doi:10.1016/j.aorn.2014.01.018

Despacho n. ${ }^{\circ}$ 1380/2018 de 8 de fevereiro. Diário da República N. ${ }^{\circ}$ 28/2018 - II Série. Gabinete do Secretário de Estado Adjunto e da Saúde. Lisboa, Portugal.

Fan, C., Pawlik, T., Daniels, T., Vernon, N., Banks, K., Westby, P., . . Makary, M. (2016). Association of safety culture with surgical site infection outcomes. American College of Surgeons, 222(2), 122-128. doi:10.1016/j.jamcollsurg.2015.11.008

Liberati, A., Altman, D., Tetzlaff, J., Mulrow, C., Gøtzsche, P., Ioannidis, J., . . . Moher, D. (2009). The PRISMA statement for reporting systematic reviews and meta-analyses of studies that evaluate health care interventions: Explanation and elaboration. PLoS Med, 6(7), e100100. doi:10.1371/journal.pmed.1000100

Molina, G., Singal, R., Haynes, A., Mahishi, V., Davis, K., Foster, G., . . Berry, W. (2017). Perception of safety of surgical practice among healthcare professionals who work in an operating room in ambulatory surgery centers in the United States: A retrospective analysis of survey data. Perioperative Care and Operating Room Management, 8, 12-18. doi:10.1016/j.pcorm.2017.07.002

Nelson, R. (2018). Ambulatory surgery centers: Are they safe? American Journal of Nursing, 118(10), 15-16. Recuperado de https://www. nursingcenter.com/pdfjournal?AID=4799481\&an=00000446201810000-00011\&Journal_ID=54030\&Issue_ID=4799451

Peters, M., Godfrey, C., McInerney, P., Munn, Z., Tricco, A., \& Khalil, H. (2020). Chapter 11: Scoping reviews. In M. Z. Aromataris (Ed.), Joanna Briggs Institute Reviewer's Manual, JBI (Cap. 11). Recuperado de https://reviewersmanual.joannabriggs.org/

Pinto, J., \& Sarnadas, L. (2020). Tradução e adaptação do Ambulatory Surgery Center Survey on Patient Safety Culture para a população portuguesa. Revista de Enfermagem Referência, 4(1), 1-10. doi:10.12707/RIV19062

Serviço Nacional de Saúde. (2020). \% de episódios GDH ambulatórios cirúrgicos para procedimentos ambulatorizáveis. Recuperado de https://app.powerbi.com/view?r=eyJrIjoiZjJiMTgyNmUtYWIxZS00MTgyLTlmNjMtYTdIYjg4N2IxY2IyIiwidCI6IjIyYzg0NjA4LWYwMWQtNDZjNS04MDI0LTYzY2M5NjJINWY1MSIsImMiOjh9

Smith, S., Sorra, J., Franklin, M., Rockville, W., \& Behm, J. (2015). Ambulatory surgery center survey on patient safety culture: User's guide. Recuperado de https://www.ahrq.gov/sites/default/files/ wysiwyg/professionals/quality-patient-safety/patientsafetyculture/ asc/userguide/ascguide.pdf 
Pinto, J. R. et al. 